Mots. Les langages du politique

$114 \mid 2017$

Le rapport, entre description et recommandation

\title{
La littérature contemporaine au rapport. Détournements d'un genre
}

Reporting in contemporary literature. Distortions of a discourse genre

El informe en literatura contemporánea. Distorciones de un género discursivo

\section{Mathilde Roussigné}

\section{(2) OpenEdition}

Journals

Édition électronique

URL : https://journals.openedition.org/mots/22843

DOI : $10.4000 /$ mots. 22843

ISSN : 1960-6001

Éditeur

ENS Éditions

Édition imprimée

Date de publication : 10 juillet 2017

Pagination : 145-163

ISSN : 0243-6450

Référence électronique

Mathilde Roussigné, "La littérature contemporaine au rapport. Détournements d'un genre », Mots. Les langages du politique [En ligne], 114 | 2017, mis en ligne le 10 juillet 2019, consulté le 22 avril 2022.

URL : http://journals.openedition.org/mots/22843; DOI : https://doi.org/10.4000/mots.22843 


\section{La littérature contemporaine au rapport. Détournements d'un genre}

On observe au pôle de production restreinte du champ littéraire français contemporain l'apparition de textes qui, à la croisée de démarches documentaires, poétiques et expérimentales, adoptent la «scénographie » du genre du rapport, pour reprendre le terme de Dominique Maingueneau (2004) dans ses travaux sur le discours littéraire ${ }^{1}$. S'ils se donnent les apparences génériques du rapport, ces textes semblent pourtant s'inscrire sur une "scène générique " tout autre, puisqu'ils relèvent explicitement ou non du poème ou du « texte de fiction ». Ainsi du texte La Base, rapport d'enquête sur un point de déséquilibre majeur en haute mer de Hugues Jallon (2004), qui débute selon le dispositif scénographique suivant:

Madame, messieurs,

Conformément à la demande que vous avez souhaité formuler, je me permets de vous adresser le rapport préliminaire exposant les premiers résultats de l'enquête sur LA BASE et les activités conduites depuis maintenant sept mois par l'Équipe spéciale placée sous ma direction. (Jallon, 2004, p. 18)

Ce « rapport» à l'ouverture très conventionnelle paraît pourtant sous le signe de l'expérimental, dans l'éphémère collection «Récifs» des Éditions du Passant qui se proposait d'accueillir «des textes résolument contemporains, tant dans leur forme graphique et stylistique que dans leur contenu, qui enquêtent sur le sens de l'ordinaire en mettant des mots là où il n'y en a pas [...] Ces textes questionneront [...] les différents modes narratifs, pourront à l'occasion se saisir du reportage et autres jeux avec le réel. „» ${ }^{2}$ Entre le conforme, c'està-dire «ce qui avait été prévu, stipulé» et l'exploration formelle, le premier contact avec La Base se situe donc précisément sur un point de déséquilibre

1. Dominique Maingueneau propose ainsi de distinguer trois composantes de la scène d'énonciation littéraire : la « scène englobante » impliquant un interdiscours préexistant aux règles de production spécifiques, la «scène générique » définie plus précisément par le genre de discours mobilisé par le texte et enfin la «scénographie », "scène de parole » singulière que le texte met en place.

2. Site Internet du Passantordinaire, 〈http://www.passant-ordinaire.com (inaccessible, 28 mars 2017).

Université de Paris 8-Saint-Denis, Littérature et histoires (EA 7322) mathilde.roussigne@gmail.com 
majeur : celui de la nature générique du texte. «Texte de fiction » ainsi que le précise la quatrième de couverture, impliquant le pacte habituel d'une suspension volontaire de l'incrédulité, il propose pourtant dès les premières lignes un pacte second - ou du moins il en prend les traits, il s'y conforme : le pacte qui lie l'auteur d'un rapport à son commanditaire.

Le rapport, genre aux contours flous, a en effet pour propriété principale d'être un compte à rendre ou un compte rendu. Se présentant à la fois comme rapport d'enquête et rapport d'activités, La Base s'attache surtout à définir une situation d'énonciation unique dans laquelle un rapporteur expose ce que l'on peut nommer sa relation à un commanditaire 3 . C'est précisément ce type de contrat que met en scène un autre texte de fiction se présentant comme poème, Le Commanditaire d'Emmanuel Hocquard (1993), qui débute par l'engagement du rapporteur :

M. Möbius, la Société que je représente m’a chargée de vous engager [...] Si vous acceptez notre proposition, vous toucherez une avance de $75000 \mathrm{~F}$, plus les frais. Nous estimons en outre que vous aurez besoin d'un pied-à-terre sur place. Je ferai les démarches nécessaires. Vous recevrez le contrat à signer dans les jours qui viennent [...] Le dossier est vide. C'est pour le remplir que nous vous engageons. (Hocquard, 1993)

Ainsi que le soulignait Todorov (1978), «chaque type de discours qualifié habituellement de littéraire a des "parents" non littéraires qui lui sont plus proches que tout autre type de “discours littéraire” ". La parenté avec le genre4 du rapport est explicite et revendiquée dans ces textes contemporains, soit qu'ils prétendent tirer leur raison d'existence de la commande d'un rapport, comme c'est le cas dans Le Commanditaire, soit qu'ils réagencent un rapport déjà existant5, soit qu'ils se présentent eux-mêmes comme rapports. Ce sont ces derniers textes qui jouent de l'hybridation générique que l'on analysera ici, textes traversés tout à la fois par les « potentialités génériques » du texte de fiction et celles du rapport, ou participant à la fois du genre du rapport et de celui du poème expérimental. L’approche dynamique selon les degrés de «généricité» des textes plutôt que selon leur appartenance à une catégorie unique (Adam, 2011) permettra de saisir la complexité de leurs fonctionnements et de

3. On s'en tiendra ici à une acception très large du genre du rapport qu'il s'agirait de préciser en fonction de critères précis (domaine d'activité professionnelle, rapport provisoire/rapport final, statut du commanditaire, etc.) qui distingueraient des sous-genres.

4. On entend ici la notion de genre dans sa définition élargie de «convention discursive». L'ensemble du corpus littéraire ici sélectionné, par la mise en place de ces situations d'énonciations fictives, confirme, si besoin est, l'existence du rapport comme véritable genre défini avant tout par des caractéristiques pragmatiques.

5. C'est le cas de Gaza d'ici-là de Frank Smith (2013) paru aux éditions Al Dante, comme le «Rapport $n^{0} 2$ » de Christophe Hanna. Le texte pousse à l'extrême la logique du «rapport déplacé »: composé uniquement de fragments d'un rapport réel de l'ONU, le Rapport de la « Mission d'établissement des faits sur le conflit de Gaza », il ne présente pas de travail de commentaire. 
leurs effets pragmatiques. Le corpus est constitué de textes marginaux dans la production littéraire française, affichant avant tout une volonté expérimentale et s'adressant à un lectorat averti, que l'on songe à La Base de Hugues Jallon, présenté comme auteur de «fictions atypiques» par les éditions Verticales, son éditeur principal, mais également aux rapports fournis par le poète Christophe Hanna sous le pseudonyme «La Rédaction ». Sous forme de contributions à des ouvrages poétiques collectifs, comme le "Rapport n 2 » dans Ouvriers vivants (1999), issu d'une rencontre avec des ouvriers sans papiers et le «Rapport n¹5b » dans La Gueule de l'emploi (1999), recueil de photographies et de poésie contemporaine publié par une École des Beaux-Arts, ou sous forme de livre pour Les Berthier, portraits statistiques (2012), il semble bien s'agir, ainsi que le revendique la collection «Réalités non couvertes » des éditions Questions théoriques dans laquelle sont parus ces portraits, d' " inventer des formes descriptives ou investigatoires, ou proposant des manières différentes de consommer les données " 6 . Si le péritexte des publications de Christophe Hanna et de Hugues Jallon affirme sans détour l'appartenance des textes au genre du rapport (titres, résumés), il faut toutefois préciser que ces derniers se conforment, empruntent leurs caractéristiques à des sphères d'activités professionnelles diverses : les services secrets du renseignement pour le directeur de l'équipe spéciale dans La Base, la police technique et scientifique travaillant sur les logiciels de portraits-robots dans le «Rapport n $15 \mathrm{~b}$ », l'Office français de protection des réfugiés et apatrides et le Haut commissariat des Nations-Unies pour les réfugiés (HCR) dans le «Rapport nº 2 », le sondage d'opinion dans Les Berthier. Ce qu'ils ont en partage, c'est leur intérêt pour cet au-dehors de la poésie qu'est la langue professionnelle, administrative, c'est leur souci d'interroger un mode de communication ordinaire de plus en plus présent, voire envahissant dans le champ des discours.

L'intérêt de l'analyse d'un corpus de rapports littéraires est alors double : en détournant le rapport de ses contextes de production traditionnels et en mettant à l'épreuve la langue qui le fonde, la littérature rend tout d'abord visibles les conventions discursives de ce dernier. Elle joue ainsi le rôle de ce que Christophe Hanna nomme la «remarque», véritable « correcteur d'attention », «acte illocutionnaire au cours duquel l'attention de l'interlocuteur est redirigée par le locuteurvers des faits contextuels insaisissables dans l'ordre attentionnel commun, propre au contexte d'interlocution » (Hanna, 2014). Par ailleurs, si ces textes se conforment de manière explicite aux principaux codes du rapport, ils ne cessent pourtant de laisser effleurer le difforme en leur sein. Autrement dit, ils s'attachent à perturber la forme mais aussi et surtout à parasiter les fonctions discursives habituelles du rapport que sont précisément la description et la prescription.

6. Page de présentation de la collection 〈http://www.questions-theoriques.com/theme/4/Realites\%20non\%20couvertes〉 (consulté le 28 mars 2017). 


\section{Compositions}

Contre l'idée reçue que le genre du rapport aurait peu à voir avec la littérature, on observe dans la production contemporaine la multiplication de textes qui brouillent les lignes de séparation départageant le documentaire de la fiction et pouvant être identifiés génériquement sous le nom de «fictions documentaires» dont le principe est d' « entrecroiser différents types de documents réels pour élaborer des sens inédits» (Pireyre, 2014). Il s'agit moins pour ces écrivains de travailler sur la langue comme matériau pur que de composer avec ce qu'Olivier Cadiot et Pierre Alfieri nomment des «Objets littéraires non identifiés» (OLNI), bousculant ainsi les conceptions communes du poème comme objet langagier esthétique et autotélique. En effet, objets déjà manufacturés, résultant d'un travail, ces derniers peuvent «aussi bien être des trouvailles que des lieux communs, aussi bien des agglomérats inédits que des bouts surcodés, aussi bien une bizarrerie ou un accident syntaxique qu'une phrase morte qu'on exhume» (1995). À ce titre, les routines langagières du rapport, les cadres de présentation et de composition qu'il propose, les phrases mortes ou la langue de bois qu'il donne parfois à lire, sont autant d'OLNI dont la littérature contemporaine s'empare avec la volonté d'explorer les possibilités offertes par le langage standardisé.

Ce souci de composer à partir de textes déjà présents, déjà donnés, est clairement affiché dans le «Rapport no 2 » et le «Rapport n $15 \mathrm{~b}$ » de La Rédaction, qui précise dans la fiche de présentation qui précède chaque rapport:

\section{La Rédaction évite l'écriture.}

L'activité déclarée de La Rédaction consiste en ce qu'elle rédige.

La responsabilité de l'écriture étant laissée aux seuls agents de La Rédaction : tout document comme tout écrit d'agent sera d'abord l'objet d'examens spécifiques, soumis ensuite au crible des processus d'analyse rédactionnels pour aboutir en dernière phase à l'information rédigée. Généralement, l'écriture n'intéresse La Rédaction que comme activité productrice d'indices. (Hanna, 1999)

L'abandon du projet d'écriture au profit d'une entreprise d'agencement par écrit invite d'ailleurs le théoricien Jean-Marie Gleize (2009) à qualifier une grande partie de ces textes de "post-poétiques», textes qui se situent en dehors de la poésie7 et «n'obéissent à aucune intention esthétique particulière, ne se réfèrent à aucun système de valeur esthétique, conventionnel ou moderniste».

7. Jean-Marie Gleize précise cependant : «ce qui ne signifie pas que l'institution ne les y reconduise sans cesse, la déclaration de la sortie de la poésie, même assortie d'une pratique confirmant cette déclaration, ne suffit pas à modifier les grilles de la réception sociale, celles de l’École, de la Bibliothèque, de la Librairie, etc. » (2009). 
43

Je joins cette correspondance de adressée de Christmas Island), l'une des dernières dont nous disposons avant son transfert qui accrédite ce qui précède concernant le rôle d' 'Bthan:
[Correspondance de [0] ] (confidentiel, adressé au SG)

"Je sais maintenant qu'il avait, depuis le premier jour à Amsterdam, tous les moyens à sa disposition, financiers notamment, pour organiser sa dissidence complète et définitive. Les éléments que nous avons reçus par la suite, les indices qu'il a semés, prouvent qu'il souhaitait - j'ignore dans quel but - que nous suivions la faible trace qu'il laissait derrière lui. "

Figure 1 - Hugues Jallon, La Base (2004, p. 96-97)

Leur principe directeur étant celui d'une «combinaison de données ou de contenus de savoirs collectés ici ou là ", l'hétérogénéité et la fragmentation textuelles sont les premières caractéristiques de ces fictions documentaires: que l'on songe par exemple au «Rapport nº 2 » de La Rédaction qui agence fiches de présentation, notes descriptives, extraits de consignes concernant l'attitude professionnelle, déclarations d'un requérant, tableaux de données, résumé de récits, retranscriptions d'entretiens, etc. Le texte La Base quant à lui se compose de courts fragments juxtaposés d'une page en moyenne numérotés de 1 à 49 et entrecoupés de multiples documents insérés, issus de la Synthèse des conclusions de l'enquête conduite par l'agent Joy (notés «SCE Joy 1 »), du journal de l'agent Ethan ou encore de correspondances confidentielles. Les documents ainsi insérés sont autant de ruptures formelles - puisque la police ou encore la couleur des pages sont modifiées - que scénographiques - puisque ces inserts proposent de nouveaux dispositifs énonciatifs en empruntant par exemple au genre du journal ou de la lettre.

La Base a pourtant ceci de particulier que ces matériaux à partir desquels elle compose sont eux-mêmes de pures fictions qui se conforment avant tout à l'hétérogénéité et la fragmentation textuelles attendues dans un rapport. La relation hypertextuelle entre $L a B a s e$ et le genre du rapport relève ainsi de l'imitation, pour reprendre la terminologie genettienne ${ }^{8}$.

Le goût pour les jeux compositionnels qui caractérise ces rapports est ainsi à saisir sous l'éclairage d'une double influence : influence de la littérature

8. Gérard Genette (1982) distingue deux modes de relations hypertextuelles : la «transformation » qui «s'en prend à un texte » spécifique et l' «imitation » qui reproduit un style, une manière. 
contemporaine et des fictions documentaires qui font du montage d'objets dissemblables l'un de leurs dispositifs privilégiés, mais influence également de l'image attendue du rapport lui-même fortement marquée par l'hétérogénéité textuelle et la juxtaposition de textes, documents, images. De même que Jean-Michel Adam (2011) souligne la « polyfonctionnalité des marques », c'està-dire la possibilité pour un schéma syntaxique de structurer des genres absolument différents, on peut ici interroger la similarité des patrons compositionnels du rapport et des fictions documentaires. Grâce à ce pont entre les deux genres, «l'image du texte» (Souchier, 1998) propre au rapport peut ainsi être conçue comme terrain d'enquête idéal pour la poésie contemporaine et son projet d'exploration textuelle.

Le «Rapport n⿳015b » de La Rédaction, paru dans l'ouvrage collectif au titre significatif La Gueule de l'emploi, se présente comme une «observation pratique des notions : portrait / ressemblance / expression / perspective » à partir d'une enquête sur le logiciel de production de portraits-robots RIPPS (Reflex Identification Portrait Provider System). Ce nouveau logiciel, indique le rapport, est «l'indice patent d'une rupture dans la façon de produire et de percevoir les figurations picturales»; en effet, il ne vise pas à représenter aussi fidèlement que possible le visage réel du criminel à partir du témoignage de la victime, mais plutôt à construire une base de données des meilleures traductions graphiques à associer aux expressions verbales dominantes chez chacun de nous. Ainsi, en retenant dans les dépositions, affaire après affaire, les traductions graphiques qui se sont avérées les plus ressemblantes, le portrait n'est certes pas ressemblant au sens photographique mais il devient efficace - la vitesse d'identification étant le critère de ressemblance retenu par les services de police. Il a pour unique but de provoquer chez l'observateur ultérieur des «identifications réflexes » et d'atteindre un «réalisme statistique optimum ». En d'autres termes, il n'a pas à être fidèle au réel mais à la représentation que la majorité peut s'en faire.

Le sujet-même du rapport choisi par La Rédaction nous semble particulièrement important au sens où il met en abyme le fonctionnement du texte luimême et son lien avec le genre du rapport : il s'agit moins, pour l'ensemble des rapports littéraires de notre corpus, de ressembler à ce qu'est réellement un rapport, que de ressembler à l'imaginaire consensuel que le lecteur s'en fait, et ce en adoptant le même principe d'efficace que celui des portraits robots : celui de l'identification réflexe. Ainsi peut s'analyser le travail formel de typographie, d'encadrement et d'encarts multiples, de fléchage, d'insertion d'images, de photographies et schémas (fig. 2).

Le «Rapport n 15b » cherche à avoir « la gueule de l'emploi », et ce presque à outrance, rendant ainsi visibles, portant notre attention notamment sur les procédés d' «énonciation éditoriale » (Souchier, 1998) qui visent à faire autorité, que l'on pense par exemple au logotype «L. R. », estampille de taille exces- 


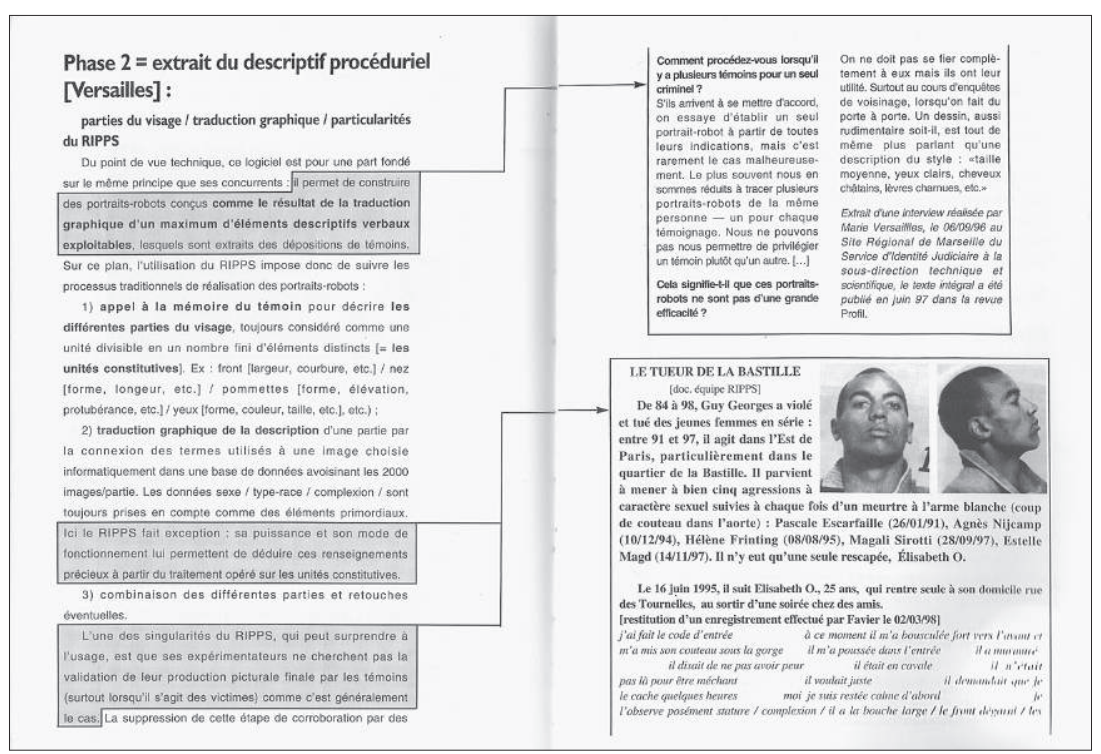

Figure 2 - La Rédaction, « Rapport nº 15b » (Hanna, 1999a)

sive, quasi-hypnotique, qui trône sur la première page, ou encore aux très nombreuses expressions inscrites en caractères gras - phénomène typographique très rare dans les publications littéraires, qui aiguillent la lecture, réduisent les marges de liberté du lecteur en lui indiquant l'information principale. Il en est de même dans La Base concernant l'apparition des noms des agents : leur insertion sur des documents caviardés et par une police de type machine à écrire évoque plus l'imaginaire de la mission secrète de renseignement que les réelles compositions du rapport d'enquête.

La lecture du «Rapport n¹5b » est également fléchée grâce à un travail de disposition textuelle qui oppose, sur les pages de gauche, le contenu principal (les écrits des agents) et sur les pages de droite l'entreprise « rédactionnelle» qui donne à lire et à voir des documents supposés résonner avec certains contenus sélectionnés. La relation fonctionnelle entre le contenu principal et les documents varie cependant en fonction des différentes étapes du «Rapport n $15 b$ », organisé explicitement en trois phases. Dans les deux premières, ainsi que le rappelait la fiche de présentation de La Rédaction, «chaque texte est analysé [...] comme un tissu d'indices ». Les documents insérés sur la page de droite ont alors pour caractéristique principale d'opposer du particulier au discours général et abstrait des extraits de rapports et du descriptif procédural, tissus d'indices que les documents démêlent, éclairent : photographie des travailleurs et de leurs ordinateurs, extrait d'une interview de l'un d'entre eux sur le site régional de Marseille du Service d'identité judiciaire, étude du cas du criminel Guy 
Georges et du manque de ressemblance de son portrait-robot (cf. Fig.2), retranscription de la déposition de sa victime, avis d'un membre de l'équipe RIPPS...

Chaque document est une véritable clef de lecture pour le non-spécialiste. Il lui donne tout d'abord accès au terrain (à la réalité médiatisée par l'enregistrement, la retranscription ou la prise photographique) : la plupart des documents parlent, donnent à voir et à entendre le «je » ou le «nous» des acteurs, travailleurs, victimes, et semblent ainsi participer de l'entreprise de description objective du logiciel. Cependant, les choix d'articulation des documents au texte révèlent aussi et surtout le rôle de faire-valoir pour le logiciel que représente le document. Celui qui développe le cas de Guy Georges, par exemple, permet de comprendre la difficulté que posent les données «type-race» et de là valorise les avancées du logiciel RIPPS qui «fait exception » dans ce domaine. Le document renoue alors ici avec son origine didactique (docere), il renseigne mais surtout il enseigne, oriente tout en jouant le rôle de preuve.

Dans la troisième phase qu'annonçait la fiche de présentation de La Rédaction pourtant, celle de «l'information rédigée » et des «commentaires rédactionnels », on observe un changement des relations entre écrits principaux et documents qui met en question les fonctions habituelles de ces derniers. Citation d'un historien de l'art, extrait de conférence d'un membre de RIPPS sur les notions de ressemblance et de réel, extrait de rapport sur un logiciel d'échecs, le document se fait ouverture théorique, support principal pour la méditation du lecteur, et va jusqu'à envahir l'espace du texte principal lors du développement sur le cas Hall, véritable réflexion sur la signification du portrait-robot qui ne représente peut-être que la folie de celui qui le produit. «Un portrait-robot, c'est représentatif de quoi ? » titre l'un des documents, tandis qu'Erwin Panofsky nous apprend que «la philosophie médiévale ne conçoit pas les œuvres d'art en relation avec un objet naturel qu'elles chercheraient à imiter, mais en relation avec le processus de formation par lequel elles accèdent à l'être».

D'un point de vue pragmatique, le dispositif s'infléchit donc progressivement : il ne s'agit plus ni de saisir objectivement les potentialités et les limites du logiciel RIPPS, ni d'en vanter les avancées techniques à un commanditaire intéressé. Le rapport est bien publié dans une maison d'édition artistique - les éditions de l'École régionale des Beaux-Arts de Valence (Drôme), dans un recueil de textes de poésie expérimentale, ainsi les documents ne jouent plus le rôle d'éclairages précis ni de preuves, même s'ils en conservent la forme, la mise en page. Ils ne jouent pourtant pas non plus clairement le rôle de signal méta-discursif, indice pour le lecteur de la nécessité d'adopter une posture interprétative devant un texte qui serait une pure réflexion sur sa propre langue. En intégrant au rapport un questionnement sur le «langage majoritaire du bon sens» et les processus d' "identification réflexe», La Rédaction sème seulement le trouble, perturbe le lecteur à un niveau «infra-herméneutique» caractéristique de ce type de textes selon Thomas Mondémé (2003). Elle met 
en doute, presque insidieusement, la première lecture-réflexe que tout lecteur adopte face à ce texte «portrait-robot» de rapport.

Cette relation hypertextuelle de type transformationnel (Genette, 1982), puisqu'il s'agit de modifier la fonction du document à partir d'un rapport préexistant, oscille entre les régimes sérieux et ludique. S'agit-il d'une simple augmentation visant à prolonger les réflexions du rapport par l'ajout de commentaires rédactionnels et de documents annexes? Ou s'agit-il plutôt d'une dénaturation parodique de la hiérarchie attendue entre textes et documents? Et de là, sous quel contrat de lecture aborder les derniers documents, à savoir la transcription d'une déposition de victime par un logiciel et un schéma sommaire du fonctionnement de la ressemblance? Le texte semble cumuler les deux effets discursifs : il suscite à la fois fascination devant la science - les enjeux des avancées techniques mais aussi la manière documentée de les rapporter - et sentiment d'étrangeté suite aux interférences.

Avec en héritage les pratiques du ready-made et du cut-up, les rapports littéraires, parce qu'ils se présentent comme des séries de coupes et de montages d'énoncés déplacés, déstabilisent donc la lecture selon deux procédés : l'exhibition de l'hétérogénéité qui leur est propre et le dérèglement pragmatique par le déplacement du rapport en contexte discursif littéraire. Mais ils ajoutent à ces pratiques habituelles9 un travail d'écriture pour Hugues Jallon, de commentaire rédactionnel pour Christophe Hanna, qui est fondamental dans leur entreprise de monstration, voire de déstabilisation discursive.

\section{Figements}

Les textes littéraires, s'ils s'emparent du genre du rapport, s'attachent à être conformes à l'«image » mais également à la langue qui le caractérise, mettant au jour les figements et la langue de bois des écrits professionnels standardisés. On observe d'emblée dans La Base le souci de rendre visibles certaines unités figées par le procédé de la citation, qui, en modifiant la prise en charge énonciative, met à distance autant qu'en relief les éléments de langage ainsi isolés. Le début du texte est criblé d'expressions dont le narrateur se dédouane clairement :

vous avez souhaité que l'Équipe spéciale inscrive sa réflexion et son action dans une logique «ouvertement proactive» (Jallon, 2004, p. 21);

ce que vous avez désigné comme leur «exceptionnelle sensibilité au risque» (Jallon, 2004, p. 21) ;

ce qu'ils appellent vulgairement un «monde instable et complexe» (Jallon, 2004, p. 27) ;

9. On référera notamment, pour plus d'informations, à l'ouvrage de Gaëlle Théval (2015). 
La citation repose ici sur un procédé discursif de distanciation critique proche de l'ironie : le locuteur mentionne des unités linguistiques et sémantiques dont il ne prend pourtant pas la responsabilité énonciative. Le discours se structure donc de manière polyphonique : il implique à la fois un locuteur rapportant des énoncés et des énonciateurs («vous» et «ils») à qui le locuteur attribue la responsabilité de ces énoncés ${ }^{10}$. Par cette mise en scène énonciative, le locuteur organise ainsi un conflit de points de vue, refusant de prendre en charge ces énoncés dont il souligne d'ailleurs le figement en les prolongeant :

Les «compétences particulières», intellectuelles physiques psychologiques, des membres (inspecteurs, enquêteurs, agents ordinaires, spéciaux, opérateurs, animateurs, comme on voudra). (Jallon, 2004, p. 29)

L'absence de virgule entre les qualificatifs accentue l'effet langue de bois, les adjectifs sont inséparables, figés dans l'apposition, tandis que l'énonciateur indique également l'aspect interchangeable des mots employés en déclinant un panel de noms à choisir à l'envi - on retrouvera ce même usage de la parenthèse quelques lignes plus bas : « des collectifs (ou équipe, cellule, staff, section, unité ) » (Jallon, 2004, p. 29).

L'exhibition de l'interchangeabilité des mots gelés est prolongée par l'apparition récurrente de ce que l'on peut nommer un "patron syntaxique " $^{11}$ : le mode binaire invasif d'apparition des noms ou des adjectifs : "principes de liberté et de tolérance », "l’idée désormais commune et partagée que notre présent et notre avenir », «l'architecture pacifique et transparente », «que l’Équipe spéciale inscrive sa réflexion et son action », « identification de signaux faibles - et moins faibles », "formules ouvertes d'étude et d'action», «modalités de recrutement parallèles et confidentielles», "indétermination relative des postes et des statuts »... (Jallon, 2004, p.19-25).

La coordination semble être de deux types : soit elle oppose deux notions complémentaires (le présent et l'avenir, l'action et la réflexion, l'étude et l'action), soit elle unit deux mots qui ont la même signification. Par ces alliances stéréotypées, le rapport littéraire rend visible le principe de fonctionnement d'un langage qui tire son autorité de l'illusion de stabilité propre aux alternances binaires, mais dont le contenu informationnel est quasi-nul. La capacité de la langue du rapport à dire quoi que ce soit va d'ailleurs jusqu'à être questionnée à l'échelle phrastique :

10. On reprend ici la distinction linguistique de Ducrot (1984) entre le «locuteur» qui organise l'énonciation et les "énonciateurs », instances discursives porteuses de différentes «voix», de différents «points de vue».

11. «Moule syntaxique avec une combinatoire lexicale plus ou moins restreinte. Les patrons peuvent être de l'ordre du syntagme ou de la proposition (patron syntaxique), voire avoir un empan inter-propositionnel (patron séquentiel)» (Née, Sitri et Veniard, 2014). 
Les sommes engagées et le temps déjà considérable consacrés à cette enquête nous ont heureusement permis de clarifier une situation dont vous avez su à l'époque mesurer les menaces que sa pérennisation pouvait faire peser sur le fonctionnement de nos existences, ainsi que le risque de déstabilisation qu'elle faisait courir aux principes de liberté et de tolérance, aux organisations internationales chargées de garantir leur respect et d'assurer, dans le cadre de leurs missions, les formes de la stabilité globale nécessaire à la poursuite de votre activité. (Jallon, 2004, p.19)

Véritable exercice de style ${ }^{12}$ à l'entrée du texte (nous sommes ici au fragment $n^{\circ} 2$ ), la phrase s'enfle dans un emboîtement de propositions plus générales les unes que les autres. La littérature met ainsi en question, par le pastiche, les capacités descriptives du rapport : les figements de la langue de bois sont autant de «routines descriptives» (Née, Sitri et Veniard, 2014) dont les effets pragmatiques dans ce contexte de réception détourné sont inversés : loin de faciliter la compréhension, ces motifs rigides au début du texte sont autant d'épreuves pour le lecteur qui peine à identifier les réalités auxquelles le discours peut référer. Le brouillage est d'ailleurs accentué par un savant mélange des styles qui emprunte autant à l'aménagement des territoires («risques sousjacents de fragilisation considérable qu'elle implique sur le maillage complexe des territoires laissés à leur soin ») qu'à l'imaginaire des services secrets ("ils ont opéré en dehors de toute procédure et organigramme officiels et n’ont rendu compte qu'au Secrétaire général »).

Si la fonction descriptive du rapport est mise à mal dans La Base, la fonction évaluative l'est tout autant. Le commanditaire a pourtant demandé, rappelle le préambule, «l'élaboration de recommandations sur la base d'analyses prospectives fondées sur une échelle consolidée des incertitudes graduée de "potentiellement certain", "difficilement probable", "certainement invraisemblable”, à "exceptionnellement possible" "(Jallon, 2004, p. 21). La charge ironique de l'effet citation, une fois encore, met en doute les capacités de la langue à pouvoir dire quoi que ce soit du réel. Le «Rapport nº 2 » de La Rédaction interroge lui aussi les outils d'évaluation attendus dans les rapports, et notamment le principe de la notation. Les fiches de présentation comportent ainsi, en caractères gras, les évaluations suivantes :

Commentaires particuliers [sur critères expressément requis par l'administration] :

- aptitude à la communication pluriculturelle: D

- aptitude à l'écoute et à la mise en confiance : B

- auto-maîtrise des comportements subjectifs : C

[sur critères rédactionnels]

12. Ou de «stylèmes » pour reprendre la suggestion de Thomas Mondeme (2013), puisque la notion de stylème est « potentiellement capable de rendre compte de marques énonciatives ou tropologiques spécifiques à un type de discours et non repérables par des effets de désautomatisation de la langue ». 
L'absence de mention d'une échelle de notation a pour conséquence pragmatique immédiate la prise de distance critique du lecteur, qui découvre progressivement que le sujet même du «Rapport nº 2 » est celui des évaluations de la crédibilité des discours des demandeurs d'asiles. Le texte inclut ainsi un rapport concernant la méthode appliquée pour faire parler un requérant, Dialou Msoubouti, ou, pour le dire avec les collocations spécialisées du HCR, «pour obtenir du requérant des unités de récit ». Il inclut également des observations concernant le «mode d’ordonnancement des unités de récit» du demandeur, ainsi qu'une évaluation globale de sa crédibilité comprenant des notes (mauvaises) sur un barème de 24 concernant ses « réactions aux faisceaux de questions », les «fondements de sa crainte », la «cohérence interne et externe et la vraisemblance générale» du récit de sa persécution. La visée argumentative du «Rapport nº 2 » ne doit cependant pas être comprise dans le seul cadre des décisions à adopter vis-à-vis du demandeur d'asile Dialou Msoubouti. Le rapport d'évaluation effectué par l'examinatrice du HCR est en effet à lire comme une justification du refus qu'elle aura probablement fait à la demande du requérant - sur la fiche de présentation de l'examinatrice, il est précisé : «elle évalue la crédibilité des déclarations des requérants; après quoi elle décide de leur accorder ou non le statut de réfugié - elle est décideur ». Mais à ce rapport sont juxtaposés des commentaires de La Rédaction intitulés «contraintes types » qui perturbent une fois encore le message énonciatif :

\section{CONTRAINTES TYPES}

- la notion de «persécution » n'étant pas définie ${ }^{\text {conv. de }}{ }^{1951}$, la réalité de la persécution est évaluée à l'aune de la crédibilité du récit.

- votre existence au moins doit pouvoir être communicable sous la forme d'une platitude fléchable et indexable : et ce dans votre intérêt vital.

L'emploi de la seconde personne du pluriel joue le rôle d'adresse au lecteur, ce dernier fusionnant ainsi avec le requérant auquel semblent destinées ces « contraintes types». Où situer alors l'évaluation ? Quel rôle discursif attribuer à ces commentaires rédactionnels? Le premier commentaire pastiche la neutralité descriptive qui domine l'ensemble du rapport, se présente comme un simple constat accompagné d'un renvoi à la référence légale (la «Convention du 28 juillet 1951 relative au statut des réfugiés » dite «Convention de Genève »). Cependant la seconde remarque, sous le ton de la recommandation, concentre une charge ironique et critique qui met à mal l'ensemble de la méthode évaluative donnée à lire dans le rapport : dévoilant les critères sur lesquels cette méthode se fonde, elle en souligne également l'indépassable limite, voire l'imposture. Alors que la «crainte fondée » et «la persécution » restent «les critères définitoires principaux du réfugié », ces critères ne sont accessibles que via le récit qui en est fait, récit sur lequel portent tous les efforts d'évaluation. 


\begin{tabular}{|c|c|}
\hline 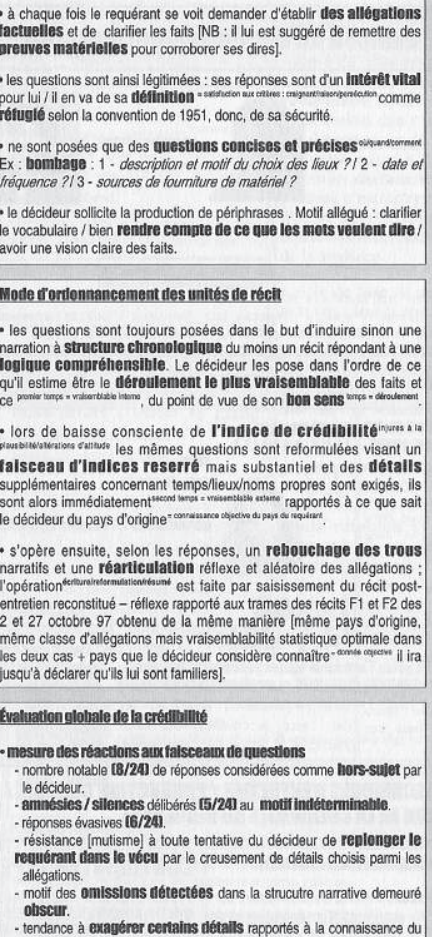 & 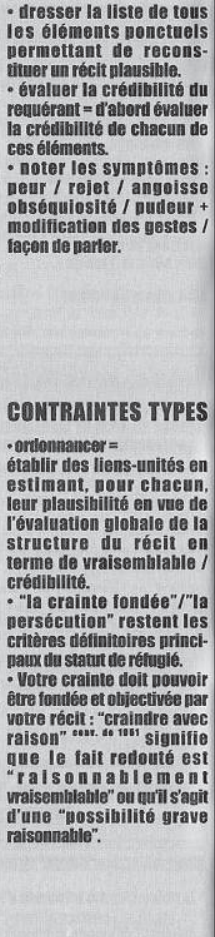 \\
\hline
\end{tabular}

Figure 3 - La Rédaction, «Rapport n०2 2 "

(Hanna, 1999b)

Soulignant l'écart entre l'«existence» et sa mise en forme "fléchable et indexable», la «contrainte type » déplace la situation d'évaluation, ou plutôt la redouble : il s'agit pour le lecteur de résister aux apparences scientifiques et objectives que fournissent les systèmes de notation et les grilles d'évaluation, d'adopter une posture critique, ou, pour le dire autrement, d'évaluer l'évaluation portée sur les demandeurs d'asile. La transformation du « Rapport no 2 » par les commentaires de La Rédaction s'effectue donc sur le mode satirique; il s'agit de dénoncer non seulement les limites des rapports des agents du HCR comme outils d'évaluation de parcours de vies, mais plus généralement les critères mêmes de l'évaluation et les conditions de régularisation des sans-papiers.

Comment penser, dès lors, la charge politique de ces discours littéraires? Faut-il uniquement les lire comme des anti-rapports qui démolissent de l'intérieur les modes de fonctionnement habituels du genre? Il est intéressant de noter que le rapport $L a$ Base comprend effectivement une partie «Recommandations » - après le «Rapport d'activité » - qui s'est finalement émancipée de "l'échelle consolidée des incertitudes» fournie par la Direction. Quelles « recommandations» les rapports littéraires ont-ils à nous livrer? 


\section{Prescriptions?}

La remise en question généralisée du modèle sartrien d'une « littérature engagée » rend complexe l'analyse du pouvoir prescriptif, d'un point de vue politique, des rapports littéraires. Le modèle pédagogique de l'efficacité de l'art pouvait être conçu de la manière suivante : l'art « expose les signes sensibles d'un certain état (disposés par la volonté de l'auteur) »; de ce fait, « reconnaître ces signes, c'est s'engager dans une certaine lecture de notre monde. Et cette lecture engendre alors un sentiment de proximité ou distance qui nous pousse à intervenir dans la situation ainsi signifiée, à la manière souhaitée par l'auteur» (Rancière, 2008, p. 58). Or précise Jacques Rancière, "le problème tient à la formule elle-même, à la présupposition d'un continuum sensible entre la production des images, gestes ou paroles et la perception d'une situation engageant les pensées, sentiments et actions des spectateurs » (2008, p. 60).

Les effets pragmatiques des textes littéraires ne répondraient donc plus à la chaîne logique «prescription-application». Christophe Hanna (2011) s'oppose d'ailleurs à la littérature dite engagée qui circule dans l'espace public et suit toujours «l'idée logocentriste d'un message envoyé depuis une position de surplomb à un destinataire, un public visé». Développant ses conceptions de la pragmatique du discours poétique, il propose de la penser selon le modèle du virus :

La poésie comme virus est un modèle d'action pragmatique camouflée par interactions de structures logiques fonctionnelles que la société tient isolées pour leur usage orthodoxe. P'3 [virus] a pour effet de provoquer à l'intérieur de ces structures un dysfonctionnement symbolique occasionnant des tensions voire des perturbations pragmatiques. P'3 [virus] n'est donc rien d'autre qu'un processus de sabotage des systèmes symboliques d'une société. (Hanna, 2004)

Procédant par dissimulation et interférences, il s'agit donc bien de créer des chocs en déplaçant le rapport en poésie et en rendant visible, par là-même, sa logique fonctionnelle.

On nuancera cependant la mise à distance radicale de la possibilité d'un «message» dans les productions poétiques de Christophe Hanna, ne seraitce que par les surenchères théoriques de l'auteur «entièrement bâties pour servir un travail d'écriture qui sans elles demeurerait invisible ou illisible ou encore mal lu » (2004), pour reprendre ses propres mots, ou par la charge critique, on l'a vu, de ses remarques rédactionnelles dans le «Rapport n 2 ». Les textes de notre corpus n'ont pas pour seule visée la déstabilisation du lecteur afin de modifier, imperceptiblement ou violemment, sa manière d'aborder les textes de rapports. La Base de Hugues Jallon notamment prétend éclairer l'histoire mal connue et bien réelle de Sealand, île-principauté et paradis fiscal, et plus généralement de zones du monde au statut juridique et à la souveraineté obscures, plate-formes de développement du capitalisme néolibéral. 


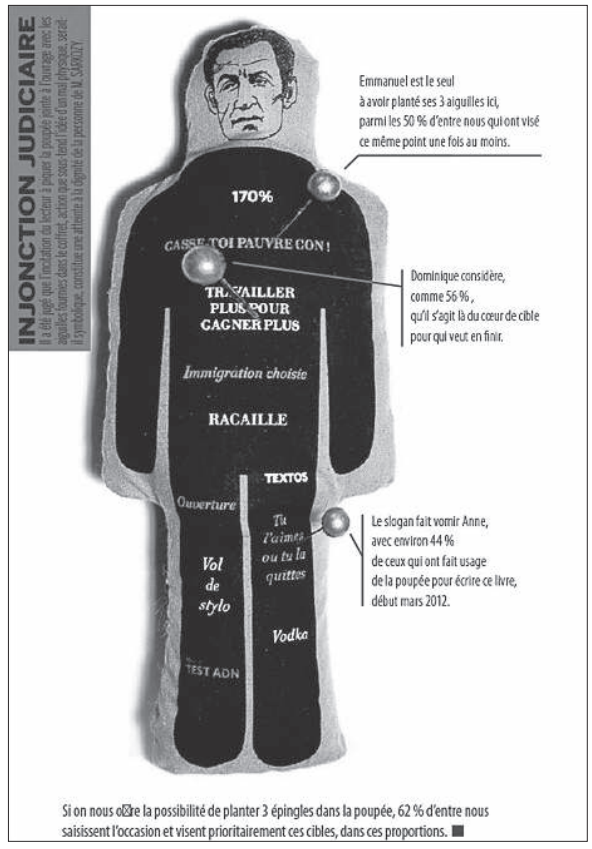

Figure 4 - La Rédaction, Les Berthier (Hanna, 2012, p.46)

Le texte Les Berthier, Portraits statistiques quant à lui est un rapport qui semble conjuguer deux intentions pragmatiques. Se présentant comme une «image statistique» de ce qui reste, dans l'opinion publique, du souvenir de la prise d'otage dans une école maternelle de Neuilly-sur-Seine par le surnommé «H. B.» en 1993, le rapport se caractérise tout d'abord par un usage détourné de la méthode statistique dans l'enquête d'opinion. Ainsi du document inséré représentant la poupée vaudou de Sarkozy, objet caricatural et satirique dont la mise en vente en France en 2008 avait donné lieu à un échec judiciaire pour le président de la République - sa demande de retrait de la poupée des magasins n’ayant pas été retenue.

"Si on nous offre la possibilité de planter trois épingles dans la poupée, $62 \%$ d'entre nous saisissent l'occasion et visent prioritairement ces cibles, dans ces proportions", indique La Rédaction (Hanna, 2012, p.46). Choix d'échantillonnage non précisé, proposition initiale explicitement orientée, la rigueur statistique est ici détournée, rappelant ainsi, comme le soulignait Desrosières (2008), que le langage mathématique, loin d'être le reflet du monde qu'il prétend être, est bien plutôt une reconfiguration du réel non exempte d'une charge subjective que la littérature prétend souligner. Le rapport utilise «l'efficacité conventionnelle des statistiques» (Bouchard, 2008) tout en en montrant les failles. 
Pour autant, il ne s'agit pas d'une simple « charge »13 contre le genre du rapport ; la publication des portraits statistiques en 2012 est à saisir en contexte. La Rédaction précise :

Nous avons vraiment commencé à le rédiger, une fois toutes les fiches faites, pendant nos vacances d'été de juillet 2011. Nous avons estimé qu'un livre ayant en librairie une "durée de vie» d'environ deux mois à partir de sa sortie, il faudrait qu'il soit terminé, relu et autorisé par nous tous, prêt à être imprimé en février 2012. Cela pour être actif en avril 2012, lorsque nous aurons à choisir notre futur président. (Hanna, 2012, p. 26)

L’appropriation et le détournement de l'enquête d'opinion prennent ainsi pleinement leur sens en contexte électoral : la prise d'otage (en 1993) ayant avant tout joué le rôle de faire-valoir à Nicolas Sarkozy, alors maire de Neuilly et ministre du Budget, il s'agit d'observer et de rappeler, sur le temps long, les effets de la manipulation médiatique dans l'opinion publique. La Rédaction ne cherche pas uniquement à déconstruire les outils de l'enquête statistique : si seul le sondage permet une saisie sur le vif, une «image » de l'opinion publique ${ }^{14}$, la littérature doit alors s'en emparer, le revisiter, en affiner les techniques et ce avec l'objectif pragmatique assumé de prendre place parmi les diverses enquêtes d'opinion qui fleurissent en pleine campagne électorale.

On retrouve donc ici l'idée du virus, d'une littérature dont l'action pragmatique est celle d'un discours camouflé. En effet, si le texte Les Berthier peut se lire comme un pastiche qui explore et détourne sur un mode ludique l'enquête statistique, il relève également de la forgerie : non seulement se présente-t-il explicitement comme un «rapport», mais il utilise réellement les outils de l'enquête d'opinion (questionnaire, calculs statistiques, etc.). Après une enquête téléphonique auprès de «quelque 130 Berthier - les Berthier de Paris, dont 102 ont répondu » (Hanna, 2012, p. 24), enquête dont le questionnaire par questions ouvertes apparaît à la fin du rapport en annexe, La Rédaction explore une nouvelle manière de décrire, de rapporter les résultats statistiques. Le texte Les Berthier ne donne pas à lire une synthèse des 102 voix sous forme de tableaux, grilles de pourcentages ou graphiques. Il fusionne plutôt le genre du rapport d'enquête d'opinion et celui du portrait littéraire, le quantitatif et le qualitatif. C'est ainsi d'un nouveau sousgenre, celui des «portraits statistiques» mentionné sur la page de couverture, dont se réclame Les Berthier, participant à la fois du genre du rapport, à un degré d'appartenance élevé, et de celui du portrait littéraire. En faisant entendre les voix personnelles de certains sondés, il rappelle constamment au lecteur la situation d'énonciation de l'entretien téléphonique et ses limites, il privilégie la nuance, ne gomme ni les incohérences qui apparaissent lors de l’entretien, ni les hésitations.

13. On entend la «charge» au sens de pratique hypertextuelle de type mimétique et satirique (Genette, 1982).

14. On réfère pour plus d'information aux travaux de Loïc Blondiaux (1998) qui montre comment la notion d'opinion publique se construit, historiquement, avec l'institutionnalisation du sondage. 
Ce souci de renouveler les modalités de description de l'opinion publique ne va pas de soi, il bouscule les dispositifs habituels de lecture de rapports d'enquêtes, ce qui relève en soi d'une visée pragmatique : ménager de nouveaux horizons d'attente chez le lecteur d'enquêtes d'opinion, qui ne se satisfassent plus du chiffré, du tranchant et du schématique. La limite de l'hybridation générique des Berthier et donc de ses effets pragmatiques réside cependant dans son contexte de production et de circulation : sa parution dans une maison d'édition de poésie d'avant-garde à la marge du champ littéraire français freine sa prétention à constituer pleinement, du point de vue de la réception, un «discours camouflé». Le degré d’appartenance générique est déterminé par la structure interne mais également par les conditions externes de parution et de réception.

Si la littérature s'approprie ainsi les codes du rapport, c'est non seulement dans le but de les rendre visibles et d'attirer notre attention sur leur fonctionnement, mais surtout pour les parasiter, c'est-à-dire s'en nourrir. Certes ces rapports littéraires, par transformation chez Hanna et imitation chez Jallon, mettent à jour les automatismes linguistiques du genre du rapport : les relations compositionnelles entre corps de texte et document sont perturbées, les figements langagiers caricaturés, les systèmes d'évaluation mis en cause. Pour autant, l'appartenance générique problématique des textes est également l'occasion d'une investigation littéraire et d'effets pragmatiques renouvelés. S'approprier le genre du rapport, c'est conformer le texte littéraire à de nouvelles «images», c'est chercher des modalités hybrides de description et d'évaluation, à la croisée de l'efficacité et de la déstabilisation.

Cherchant à brouiller la ligne de partage entre fiction et document, les textes de Hugues Jallon et Christophe Hanna s'emparent du rapport afin d'en tirer des effets pragmatiques, voire politiques. Effets en langue pour les rapports de La Rédaction par exemple : les minces modifications et commentaires visant à déstabiliser les automatismes de lecture, pour le lecteur herméneute et averti, familier des théories de Hanna. Mais il s'agit également d'affirmer la possibilité de la fiction pour Jallon, de la poésie pour Hanna, à être lues comme de véritables rapports, c'est-à-dire comme porteuses d'un savoir sur les problèmes publics. Si La Base et Les Berthier échappent ainsi à un projet autotélique, ils se heurtent néanmoins aux limites de leur contexte de production et de circulation. Les auteurs qualifient ainsi les effets pragmatiques du discours littéraire par le recours au lexique de l'infime, du minimal : "virus » chez Hanna, la fonction de la littérature chez Jallon peut correspondre au «point d'appui » évoqué dans la toute dernière phrase de la conclusion du rapport :

Je vous parle de l'assemblage patient et progressif d'une fiction lourde d'effets puissants, mais pour l'heure encore impossibles à évaluer, je vous parle d'un point d'appui discret capable à terme de soulever notre monde et les principes qui l'organisent. (Jallon, 2004, p. 111) 


\section{Références}

ADAM Jean-Michel, 2011, Genres de récits : narrativité et généricité des textes, Louvainla-Neuve, L'Harmattan-Academia.

AlfÉRI Pierre, CADIOt Olivier, 1995, La Revue de Littérature générale, 1, Paris, POL.

BLONDIAUX Loïc, 1998, La fabrique de l'opinion. Une histoire sociale des sondages, Paris, Éd. du Seuil.

BOUCHARD Julie, 2008, "La communication, le nombre, et le néolibéralisme», MEI. Médiation et information : revue internationale de communication, no 28, p. 23-35.

DESROSIÈRES Alain, 2008, «Communiquer, gouverner et être gouvernés par les chiffres. Questions à Jacques Durand et Alain Desrosières », MEl. Médiation et information : revue internationale de communication, n 28, p.7-18.

GLeIZE Jean-Marie, 2009, Sorties, Paris, Éd. Questions théoriques.

Hanna Christophe, 2002, Poésie action directe, Paris, Al Dante/L. Scheer.

HANNA Christophe, 2011, «Actions politiques / Actions littéraires», Toi aussi tu as des armes. Poésie \& politique, Paris, La Fabrique.

HanNa Christophe, 2014, "Attention et valorisation : esquisse d'une poétique de la remarque», Économie de l'attention, Y. Citton éd., Paris, La Découverte.

DuCROT Oswald, 1984, Le dire et le dit, Paris, Éd. de Minuit.

Genette Gérard, 1982, Palimpsestes, Paris, Éd. du Seuil.

HoCQUARD Emmanuel, 1993, Le Commanditaire, Paris, POL.

Maingueneau Dominique, 1998, Analyser les textes de communication, Paris, Dunod.

MAINGUENEAU Dominique 2004, Le discours littéraire : paratopie et scène d'énonciation, Paris, A. Colin.

Mondémé Thomas, 2003, "Autres pratiques interprétatives», Tracés. Revue de Sciences humaines, $\mathrm{n}^{\circ} 4$, p.69-82; disponible sur Internet <http://traces.revues. org/3873> (consulté le 28 mars 2017).

NÉE Émilie, SITRI Frédérique, VENIARD Marie, 2014, Pour une approche des routines discursives dans les écrits professionnels, Communication au Congrès mondial de linguistique française : Berlin, 19-24 juillet 2014 〈https://halshs.archives-ouvertes. fr/hal-01052318> (consulté le 26 janvier 2017).

PIREYRE Emmanuelle, 2014, "Fictions documentaires», Devenirs du roman 2 : écriture et matériaux, Paris, Éd. Inculte.

RANCIÈrE Jacques, 2008, Le spectateur émancipé, Paris, La Fabrique.

SOUCHIER Emmanuel, 1998, "L'image du texte pour une théorie de l'énonciation éditoriale », Les cahiers de médiologie, n 6, p.137-145.

Théval Gaëlle, 2015, Poésies ready-made, xxe-xxıe siècles, Paris, L'Harmattan.

Todorov Tzvetan, 1978, Les genres du discours, Paris, Éd. du Seuil. 


\section{Corpus}

HannA Christophe, 1999a, "Rapport n 15b [Les Portraits-robots] », La Gueule de l'emploi, C. Hanna, J.-L. Moulène, M. Joseph, Valence, Éd. de l’École régionale des Beaux-Arts.

HannA Christophe, 1999b, «Rapport n² 2 », Ouvriers vivants, Romainville, Al Dante.

HannA Christophe, 2012, Les Berthier. Portraits statistiques, Paris, Éd. Questions théoriques.

Jallon Hugues, 2004, La Base, rapport d'enquête sur un point de déséquilibre majeur en haute mer, Bègles, Le Passant. 